\title{
Control of spin-wave excitations in deterministic fractals
}

\author{
Christian Swoboda, ${ }^{1,2, *}$ Michael Martens, ${ }^{1}$ and Guido Meier ${ }^{1,2,3}$ \\ ${ }^{1}$ Institut für Angewandte Physik und Zentrum für Mikrostrukturforschung, Universität Hamburg, Jungiusstrasse 11, 20355 Hamburg, Germany \\ ${ }^{2}$ The Hamburg Centre for Ultrafast Imaging, Luruper Chaussee 149, 22761 Hamburg, Germany \\ ${ }^{3}$ Max Planck Institute for the Structure and Dynamics of Matter, Luruper Chaussee 149, 22761 Hamburg, Germany
}

(Received 31 October 2014; published 17 February 2015)

\begin{abstract}
We study spin-wave spectra of mesoscopic ferromagnetic Sierpinski carpets by means of broadbandferromagnetic resonance measurements and micromagnetic simulations. Sierpinski carpets are self-similar fractals with noninteger Hausdorff dimension that are constructed via a deterministic iteration process. The number of quantized spin-wave modes in the spectra increases with the iteration level of the carpets and the frequency splitting resembles bandpass characteristics known from fractal antennas. We find that the splitting is sensitive to the fractal dimension as well as to the relative alignment of the magnetic field and the sides of the fractals. Micromagnetic simulations provide the localization of individual spin-wave modes determined by the confinement and the inhomogeneity of the internal field.
\end{abstract}

DOI: 10.1103/PhysRevB.91.064416

PACS number(s): 75.75.-c, 75.30.Ds, 75.78.-n, 76.50.+g

\section{INTRODUCTION}

Spin waves are promising candidates to carry and store information in future memory applications [1-4]. In this context, the control of the properties of spin waves is in the focus of research. The spectrum of spin waves can be tuned by a geometrical or field dependent confinement that leads to a quantization into individual spin-wave modes [5-11]. Magnonic crystals [12-15] are artificial lattices providing an alternating modulation of their magnetic characteristics. The modulation can either be realized by the composition of different magnetic materials [16] or by geometrical structuring $[17,18]$. An adequate tuning of the modulation enables one to tailor band gaps in the material and to control the generation and propagation of spin waves that are limited to certain permitted frequency ranges [19]. The concept of magnonic crystals is not limited only to propagating spin-wave modes but also standing spin waves are in the focus of research [20,21].

Fractals [22] can be assigned into two different groups: random and deterministic fractals. Both these structures exhibit by definition a noninteger Hausdorff dimension [23] and can be characterized by self-similarity and scale invariance. The existence of the random type is widely spread in nature and can be found for example in Romanesque cauliflower, fern, or frost patterns. Experimental fractals investigated so far are mostly of the random type, e.g., two-dimensional deposits of dendrites at a percolation threshold exhibiting hierarchical levels obtained by diffusion-limited aggregation (DLA) [24]. Vibrational excitation spectra of percolation clusters show localized low-frequency modes named fractons [25,26]. Deterministic fractals, however, provide a self-similarity that is not statistically distributed: at every magnification a part of the structure can be found that is similar to the whole structure. Their characteristics are close to quasicrystals [27] and with respect to spin waves one can expect gaps and degeneration in excitation spectra. Well-known examples are Sierpinski carpets and gaskets. In fractal antennas these structures feature multiband and wideband characteristics [28] and can be

\footnotetext{
*cswoboda@physnet.uni-hamburg.de
}

found nowadays in cell phones. Recent numerical calculations $[29,30]$ show that spin-wave spectra of low-dimensional Sierpinski carpets are singular continuous functions of the frequency making deterministic fractals auspicious candidates for magnonic applications.

In this work, spin-wave excitations in Sierpinski carpets built from ferromagnetic nanostructures are studied by means of broadband-ferromagnetic resonance (FMR) measurements and micromagnetic simulations. The spin-wave spectra are expected to depend on the Hausdorff dimension, in the following named fractal dimension, and the connectivity properties of the fractals' subdimensions. The presentation of the experimental methods is followed by a discussion of the experimental and simulation results. The localization of the observed spin-wave modes is provided by the simulated spatially resolved magnitude of magnetization precession. In all, results for Sierpinski carpets exhibiting three different fractal dimensions are presented.

\section{SAMPLE FABRICATION}

Samples as shown in Fig. 1(a) are fabricated by electronbeam lithography and lift-off processing. By thermally evaporating $120 \mathrm{~nm}$ gold and $8 \mathrm{~nm}$ chromium as adhesive layer, a coplanar waveguide (CPW) is deposited on top of a GaAs substrate. A $200 \mathrm{~nm}$ thick layer of hydrogen silsesquioxane (HSQ) that provides a small surface roughness of $0.3 \mathrm{~nm}$ root mean square is structured by electron-beam lithography on top of the CPW. Subsequently, the Sierpinski carpets are prepared by thermal vapor deposition of $30 \mathrm{~nm}$ of Permalloy $\left(\mathrm{Ni}_{80} \mathrm{Fe}_{20}\right)$.

Sierpinski carpets $\mathrm{SC}(n, p, i)$ are constructed by a deterministic iteration process. A square is divided into $n^{2}$ congruent subsquares of which $n^{2}-p$ are removed. The resulting structure consisting of $p=n^{d}$ occupied subsquares is named the generating cell $\mathrm{SC}(n, p, 1)$ and its fractal dimension $d=\ln (p) / \ln (n)$ [31] is determined by the parameters $n$ and $p$. In other words, the fractal dimension is the ratio of the logarithm of the number of self-similar pieces $p$ and the logarithm of the magnification factor $n$. After $i$ iterations of the segmentation process that is applied on the occupied subsquares, the Sierpinski carpet $\mathrm{SC}(n, p, i)$ consists of $n^{i}$ 


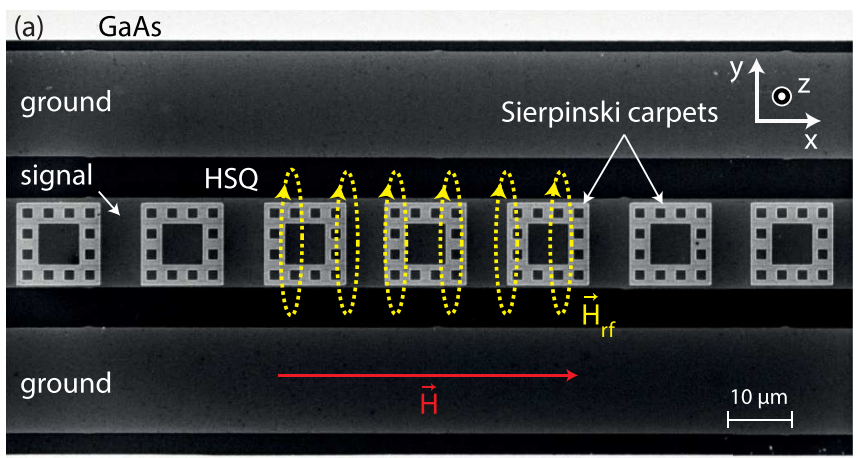

(b)

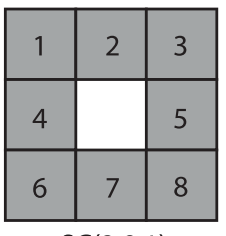

$\mathrm{SC}(3,8,1)$

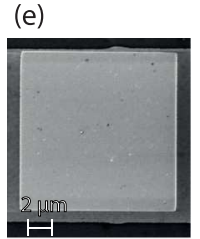

$\mathrm{SC}(4,12,0)$ (c)

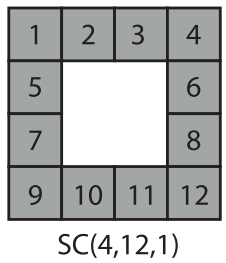

(f)

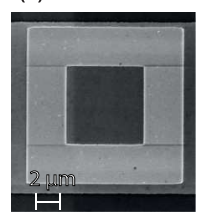

$\mathrm{SC}(4,12,1)$ (g)

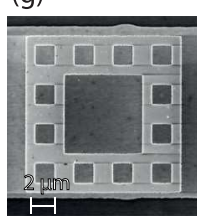

$\mathrm{SC}(4,12,2)$ (d)

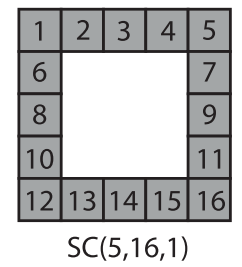

(h)

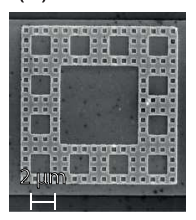

$\mathrm{SC}(4,12,3)$

FIG. 1. (Color online) (a) Scanning-electron micrograph of Sierpinski carpets $\mathrm{SC}(4,12,2)$ on a coplanar waveguide consisting of two ground and one signal lead and a layer of HSQ. (b)-(d) Generating cells of Sierpinski carpets $\mathrm{SC}(n, p, i)$ with a fractal dimension of (b) $d=\ln (8) / \ln (3) \approx 1.893$, (c) $d \approx 1.792$, and (d) $d \approx 1.723$. (e)-(h) Scanning-electron micrographs of Sierpinski carpets $\mathrm{SC}(4,12, i)$ with gradually increasing iteration levels from $i=0$ to $i=3$.

subsquares of which $p^{i}$ are occupied. Topological properties of Sierpinski carpets such as connectivity and lacunarity are defined by the geometrical location of the occupied subsquares in the generating cell [29]. In this work, the missing subsquares are exclusively located in the center of the generating cells. Figures 1(b)-1(d) show generating cells of Sierpinski carpets having different fractal dimensions.

The fractals have a size of $14 \mu \mathrm{m} \times 14 \mu \mathrm{m} \times 30 \mathrm{~nm}$ to ensure that four iteration levels are within the limits of preparation; compare Figs. 1(e)-1(h). In order to obtain a high signal-to-noise-ratio, a total of 29 to 35 fractals (depending on the sample type) are prepared on top of the CPW. The distance between the carpets is chosen large enough to exclude stray-field interactions [14,32-34] in between the individual structures.

\section{BROADBAND-FMR SPECTROSCOPY}

Experimental data are obtained by broadband-FMR spectroscopy using a vector-network analyzer [34,35]. A static external magnetic field $\vec{H}$ is aligned parallel to the CPW in the $x$ direction. The field strength $\mu_{0} H$ is varied from $-90 \mathrm{mT}$ to $90 \mathrm{mT}$. Prior to each field step, the same magnetic state is ensured by using a hysteresis field of $-90 \mathrm{mT}$. A radio-

frequency (rf) magnetic field $\vec{H}_{\text {rf }}$ is created perpendicularly to the external field $\vec{H}$ by sending a sinusoidal rf current through the signal lead of the CPW. The magnetic configuration of the experimental setup is illustrated in Fig. 1(a). For each field step $\mu_{0} H$, the radio frequency $f$ is swept between $10 \mathrm{MHz}$ and $15 \mathrm{GHz}$. Eigenfrequencies of the Sierpinski carpets are excited by energy transfer from $\vec{H}_{\mathrm{rf}}$. Thus, resonances are detected by means of a reduced transmission of the rf current through the signal lead. When applying a reference field of $90 \mathrm{mT}$ in the $y$ direction only weak excitations of edge modes [6] are expected. We measure the normalized transmission, which is the difference between transmission and reference spectrum. It allows the detection of resonance modes with a high signal-to-noise ratio.

Due to the confining geometry of the structures, we expect to exclusively excite standing spin-wave modes in our experiments. For a long stripe where external field and magnetization are aligned along the stripe's axis (easy axis), the internal magnetic field is homogeneous. However, effective boundary conditions lead to a pinning of the dynamic magnetization at the edges of the stripe and the wave vector of the spin waves is quantized along the stripe's finite width [7]. In this configuration, magnetostatic [36] Damon-Eshbach (DE) modes $[5,7,37]$ with their quantized wave vector aligned perpendicular to the magnetization are excited. When the magnetization is aligned perpendicular to the stripe axis (hard axis), the wave vector of the quantized spin waves is aligned parallel to the magnetization and the modes have magnetostatic backward volume (BV) mode character $[6,8,10]$. The internal magnetic field is strongly inhomogeneous along the stripe's width and the lowest modes are exchange-dominated modes [36] localized at the edges of the structures [6,8]. Thus, the quantization of spin waves is not limited only to the structural geometry but can also originate from inhomogeneous internal field distributions.

In thin rectangular elements with two finite in-plane sizes, there is no orientation of the external field that leads to a homogeneous distribution of the internal magnetic field. The inhomogeneity occurs mainly along the direction of the external field [11]. The two-dimensional quantization of the inplane wave vector of the spin waves [10] can be described as a product of the one-dimensional quantizations of longitudinally and transversely magnetized long stripes [11]. The quantized in-plane components of the wave vector are $k_{l x}$ and $k_{m y}$ with the indices $l, m=0,1,2, \ldots$ describing the number of nodes of the standing spin waves. In our experiments, only modes having an even symmetry about the center of the elements, i.e., modes with an even number of nodes, can be excited [38]. The inhomogeneity of the internal field leads to a localization of the eigenmodes: the lowest modes have dipole-exchange character and are strongly localized near the edges while the dipole-dominated higher modes are weakly localized near the center of the rectangles [11].

Complexity and confinement of the Sierpinski carpets increase with the iteration level. The internal field is expected to be rather inhomogeneous, especially at the edges of the holes introduced by the segmentation steps. As a result, the number of localized and quantized spin-wave modes will increase with the iteration level. Due to the strong confinement and 
the inhomogeneity of the internal field, an alignment of the magnetization that is predominantly parallel to the external field is only expected for high field strengths. For small fields, pronounced domain patterns can lead to a suppression of individual spin-wave modes [39,40] depending on their localization within the carpets.

\section{A. Dependency on the iteration level}

Figure 2 shows typical broadband spectra of Sierpinski carpets $\mathrm{SC}(4,12, i)$ having a fractal dimension of $d \approx 1.792$. The spectra of solid Permalloy squares, that are Sierpinski carpets at iteration level $i=0$, contain one prominent spinwave mode SW-01 with a frequency of $f=9.4 \mathrm{GHz}$ for a field strength of $\mu_{0} H=-90 \mathrm{mT}$; compare Fig. 2(a). The absorption line disappears at a field strength of $2 \mathrm{mT}$ indicating the switching of the magnetizations of these carpets. For positive fields, the absorption line shows a symmetric field dispersion. Above the absorption line SW-01, there are two higher-order spin-wave modes HO-01 and HO-02 of the mode SW-01, visible in the spectra for field strengths between $\pm 50 \mathrm{mT}$. Below the absorption line SW-01 there are two absorption lines corresponding to exchange-dominated modes [6], located close to the lateral edges of the square [11] in the direction of the magnetization. The differences in the field dispersion of these edge modes and the modes SW and $\mathrm{HO}$ originate from the decreased effective field at the lateral edges of the structure.

For an iteration level of $i=1$, two prominent absorption lines SW-11 and SW-12 with frequencies of $9.3 \mathrm{GHz}$ and $9.9 \mathrm{GHz}$ at $-90 \mathrm{mT}$ are visible in the spectra; compare Fig. 2(b). With decreasing field strength, the two absorption lines have different negative slopes and the frequency splitting between the lines increases up to $1.7 \mathrm{GHz}$ for a field strength of $-10 \mathrm{mT}$. The modes HO-11 and HO-12 at frequencies of 10.9 $\mathrm{GHz}$ and $11.7 \mathrm{GHz}$ for $-90 \mathrm{mT}$ are higher-order modes of SW-12 since all three modes show the same field dispersion and switch between $1 \mathrm{mT}$ and $3 \mathrm{mT}$. The intensity of SW-11 starts to decrease at $-12 \mathrm{mT}$, bends up between $-5 \mathrm{mT}$ and $-2 \mathrm{mT}$, and disappears between $-2 \mathrm{mT}$ and $6 \mathrm{mT}$ before it shows a symmetric course for positive fields. The up-bending will be discussed in Sec. V. Edge modes have the same field distribution as for solid squares $\mathrm{SC}(4,12,0)$ and are visible below the mode SW-11.

With increasing iteration level, the excitation of further spin-wave modes can be observed in the spectra. Five distinct absorption lines SW-21 to SW-25 that show a clear frequency splitting are visible in the spectra at an iteration level of $i=2$; compare Fig. 2(c). The frequencies are ranging from $8.3 \mathrm{GHz}$ for $\mathrm{SW}-21$ to $11.0 \mathrm{GHz}$ for $\mathrm{SW}-25$ at $-90 \mathrm{mT}$. The maximum values of the frequency splittings between the individual modes are $\delta f_{\mathrm{SW}-21,22}=1.8 \mathrm{GHz}$ at $-25 \mathrm{mT}$, $\delta f_{\mathrm{SW}-22,23}=1.6 \mathrm{GHz}$ at $-12 \mathrm{mT}, \delta f_{\mathrm{SW}-23,25}=1.6 \mathrm{GHz}$ at $-12 \mathrm{mT}$, and $\delta f_{\mathrm{SW}-24,25}=2.3 \mathrm{GHz}$ at $-1 \mathrm{mT}$. For small fields, the field dispersion of the individual modes is different and depends on their localization within the carpets and the local inhomogeneity of the internal field. The mode SW-25 and its higher-order mode HO-22 switch in the small field range between $6 \mathrm{mT}$ and $8 \mathrm{mT}$. The absorption line of SW-23 bends up between $-12 \mathrm{mT}$ and $-4 \mathrm{mT}$ and disappears like

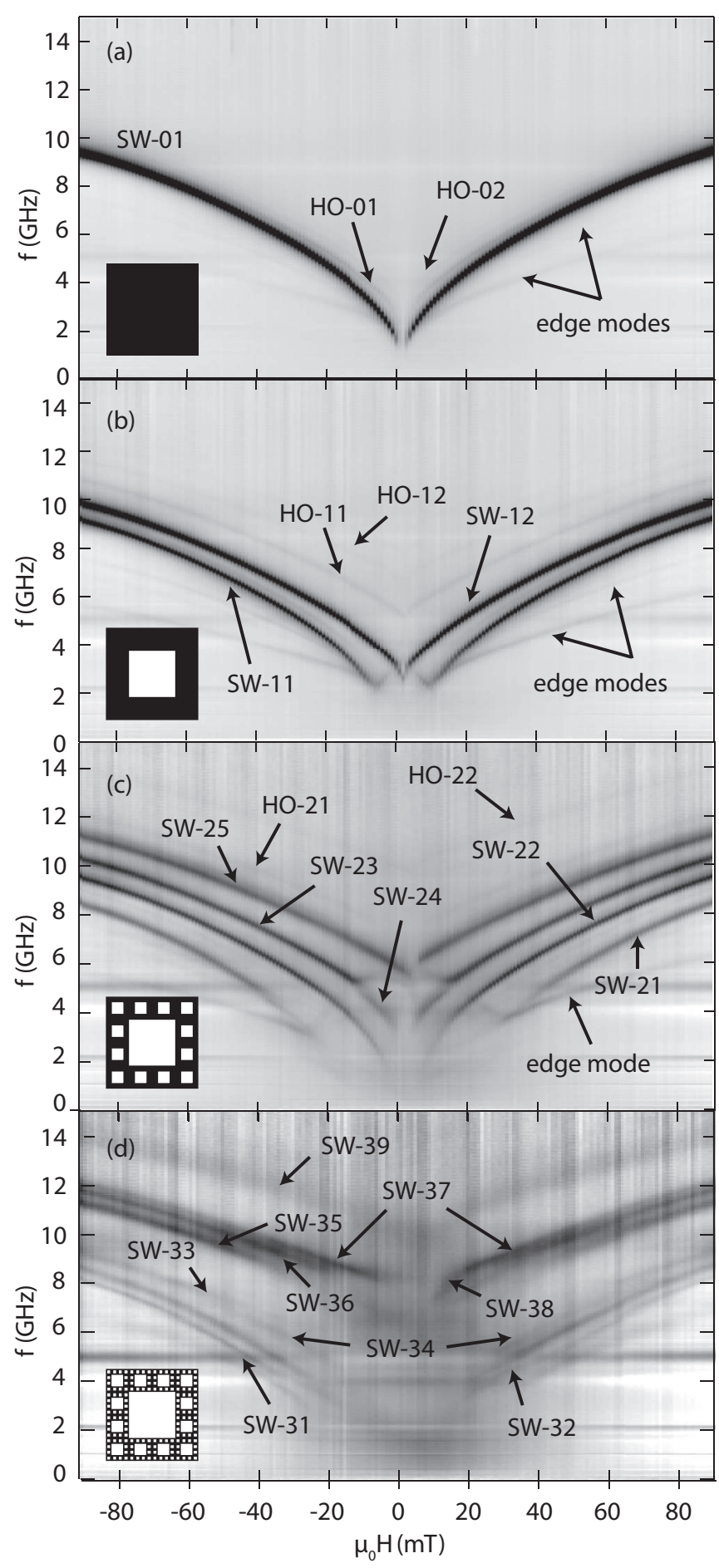

FIG. 2. Broadband-ferromagnetic transmission spectra of Sierpinski carpets $\mathrm{SC}(4,12, i)$ with a fractal dimension of $d \approx 1.792$ in dependence on the applied field strength $\mu_{0} H$. Dark contrast corresponds to a reduced transmission. The iteration level of the fractals is increased from (a) $i=0$ to (d) $i=3$ as indicated by the insets in the spectra.

the associated higher-order mode HO-21 until $8 \mathrm{mT}$. Between $-10 \mathrm{mT}$ and $-1 \mathrm{mT}$ as well as $5 \mathrm{mT}$ and $14 \mathrm{mT}$ the mode SW-24 with a similar dispersion as SW-23 appears. The absorption line of SW-22 shows a decreased intensity from 
$-21 \mathrm{mT}$ and vanishes between $-4 \mathrm{mT}$ and $9 \mathrm{mT}$. The mode SW-21 disappears between $-34 \mathrm{mT}$ and $35 \mathrm{mT}$.

For the iteration level $i=3$, nine different absorption lines are visible in the spectra; compare Fig. 2(d). The signal-to-noise ratio is significantly decreased compared to lower iteration levels because the Sierpinski carpets consist of considerably less ferromagnetic material. A first branch of the modes SW-31 to SW-34 is located between frequencies of 9.3 GHz and $3.1 \mathrm{GHz}$, a second branch of SW-35 to SW-38 between $12.1 \mathrm{GHz}$ and $8.9 \mathrm{GHz}$, and the mode SW-39 between $14.1 \mathrm{GHz}$ and $9.7 \mathrm{GHz}$. The intensity of the modes in the second branch is about three times higher compared to the remaining modes. While the frequency splitting between the individual modes in each of the two branches stays about the same for all field strengths, the splitting between the first and the second branch reaches its maximum of $2.5 \mathrm{GHz}$ at $-34 \mathrm{mT}$. The field range where individual modes disappear is extended due to the additional confinement. The mode SW-39 with the smallest gap vanishes at $13 \mathrm{mT}$ and reappears at $20 \mathrm{mT}$ while the mode SW-33 with the largest gap disappears between $-49 \mathrm{mT}$ and $61 \mathrm{mT}$. In the frequency range of SW-33, the mode SW-34 appears at $-41 \mathrm{mT}$ up to $-19 \mathrm{mT}$ and reappears at $27 \mathrm{mT}$ until $55 \mathrm{mT}$. During disappearance of SW-37, the mode SW-38 appears only for positive fields between $11 \mathrm{mT}$ and $19 \mathrm{mT}$. General features such as the increased frequency splitting between the modes and the increased complexity of the carpets can be nicely seen at this level of iteration. Here a total of 144 subsquares are removed additionally from each Sierpinski carpet and the spin-wave spectrum is rather complex. In order to sustain clarity, we focus in the further investigation on iteration levels of $i=1$ and 2 where distinct modes can be observed.

\section{B. Dependency on the fractal dimension}

In order to observe the dependence of the spin-wave spectra on the fractal dimension, Sierpinski carpets $\mathrm{SC}(3,8, i)$ and $\operatorname{SC}(5,16, i)$ with a higher and a lower fractal dimension are studied, respectively. The generating cells are given in Figs. 1(b) and 1(d). The in-plane sizes of the generating cells are the same as for $\mathrm{SC}(4,12, i)$. The fractal dimension determines the size of the holes introduced in the segmentation process while the shape of the generating cells remains unaffected; compare Figs. 1(b)-1(d). For the same magnetic configuration, we expect a change in the frequencies of the individual spin-wave modes while their location within the carpets is assumed to be similar for all fractal dimensions. Because of this correspondence we can use the same denotation for the individual modes identified by their field dispersion.

For the iteration level $i=1$ the spectra for all three fractal dimensions show the two absorption lines SW-11 and SW-12; see Figs. 2(b), 3(a), and 3(b). In comparison with Sierpinski carpets $\mathrm{SC}(4,12,1)$, a change in the maximum frequency splitting between the modes can be observed. While for $\mathrm{SC}(3,8,1)$ the splitting of $\delta f=1.3 \mathrm{GHz}$ at a field strength of $-5 \mathrm{mT}$ is $0.4 \mathrm{GHz}$ smaller, the gap is $0.4 \mathrm{GHz}$ larger for $\mathrm{SC}(5,16,1)$ with $\delta f=2.1 \mathrm{GHz}$ at $-10 \mathrm{mT}$. The confinement of the modes increases with decreasing fractal dimension and can be observed in the spectra during magnetization reversal. The mode SW-12 disappears between $1 \mathrm{mT}$ and $3 \mathrm{mT}$ for

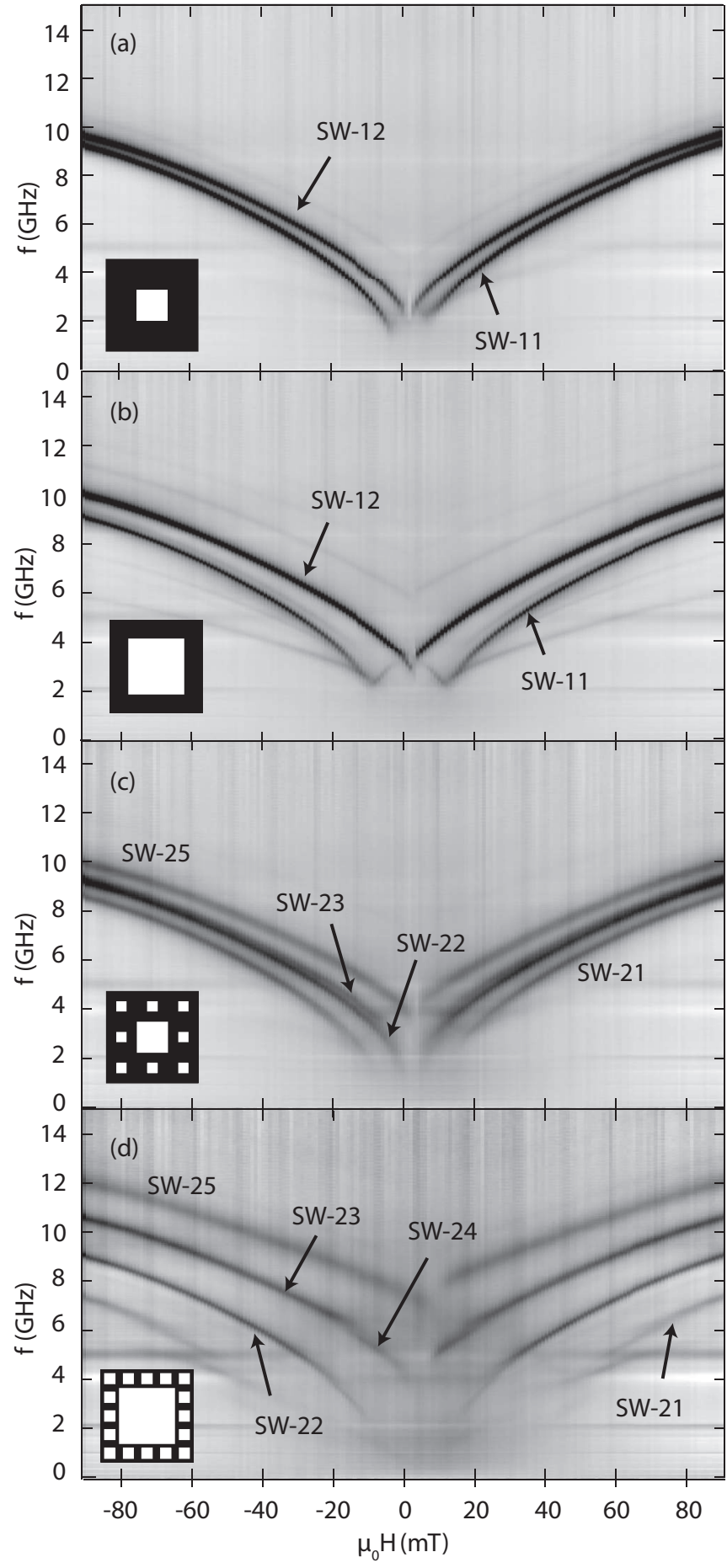

FIG. 3. Transmission spectra of Sierpinski carpets with different and additional fractal dimensions. (a) and (c) Spectra of $\operatorname{SC}(3,8,1)$ and its higher iteration level $\operatorname{SC}(3,8,2)$ with $d \approx 1.893$. (b) and (d) Spectra of $\operatorname{SC}(5,16,1)$ and $\operatorname{SC}(5,16,2)$ with $d \approx 1.723$.

$\mathrm{SC}(3,8,1)$ and switches with a discontinuity in the absorption line at $4 \mathrm{mT}$ for $\mathrm{SC}(5,16,1)$. The up-bending of SW-11 is more pronounced for $\operatorname{SC}(5,16,1)$ compared to $\mathrm{SC}(4,12,1)$ and occurs already between $-9 \mathrm{mT}$ and $12 \mathrm{mT}$. For $\mathrm{SC}(3,8,1)$, $\mathrm{SW}-11$ vanishes between $-1 \mathrm{mT}$ and $4 \mathrm{mT}$ and only a weak up-bending can be observed at $-3 \mathrm{mT}$. 
For the iteration level $i=2$, four absorption lines SW-21 to SW-25 with frequencies ranging from $10.0 \mathrm{GHz}$ to $2.0 \mathrm{GHz}$ can be observed for $\mathrm{SC}(3,8,2)$; compare Fig. 3(c). The absorption lines SW-22 and SW-23 with the highest intensities are superimposed for high field strengths and separated between field values of $\pm 25 \mathrm{mT}$. No excitation of SW-24 can be observed. The maximum frequency splittings between the modes SW-21 to SW-25 are $0.5 \mathrm{GHz}, 1.1 \mathrm{GHz}$, and $1.0 \mathrm{GHz}$ smaller compared to Sierpinski carpets $\mathrm{SC}(4,12,2)$. For the carpets $\mathrm{SC}(5,16,2)$, five absorption lines including SW-24 are visible and the frequencies are ranging from $2.5 \mathrm{GHz}$ to $12.0 \mathrm{GHz}$; see Fig. 3(d). The frequency splittings between the modes are about $0.8 \mathrm{GHz}$ larger compared to $\mathrm{SC}(4,12,2)$.

Our experimental finding is that the frequency splitting between the spin-wave modes increases with decreasing fractal dimension. This observation complies with numerical calculations of the spin-wave spectra of Sierpinski carpets. In the calculations of Monceau and Lévy [29], frequency spectra of the normalized integrated density of states (NIDOS) are singular continuous functions where gaps and plateaus in the spectra are connected to symmetry and degeneracy of the eigenmodes. With decreasing fractal dimension the authors calculate larger gaps in the NIDOS which can be associated with larger gaps in the frequency spectrum [29]. This is in agreement with our experimental findings.

\section{Variation of the external field vector}

In order to study the angular symmetry of the mode spectrum, the angle $\alpha$ between the external field vector $\vec{H}$ and the sides of the fourfold-symmetric Sierpinski carpets is varied between $0^{\circ}$ and $45^{\circ}$ in steps of $5^{\circ}$. Maximum spin-wave excitation is guaranteed by perpendicular alignment between the external field $\vec{H}$ and the rf field $\vec{H}_{\mathrm{rf}}$. Thus, instead of rotating the external field with respect to the CPW, individual samples are prepared for each rotation angle $\alpha$.

The transmission spectra of Sierpinski carpets $\operatorname{SC}(4,12,2)$ for different angles $\alpha$ are shown in Fig. 4. Between $0^{\circ}$ and $10^{\circ}$, the field dispersion of the modes SW-21 to SW-25 remains qualitatively the same. For $\alpha=10^{\circ}$, the absorption line SW-21 has a decreased intensity, shifts up by $0.2 \mathrm{GHz}$, and vanishes between $-52 \mathrm{mT}$ and $60 \mathrm{mT}$. A new mode SW-20 with a smaller slope than the remaining modes appears between $f=7.6 \mathrm{GHz}$ and $f=4.5 \mathrm{GHz}$; compare Fig. 4(a). SW-20 bends up at $-36 \mathrm{mT}$, meets SW-22 at $-22 \mathrm{mT}$, and disappears up to $27 \mathrm{mT}$. With increasing angle $\alpha$ a reduction of the complexity of the modes is observed. While SW-20 disappears at $20^{\circ}$, the intensity of SW-21 decreases continuously until complete disappearance at $25^{\circ}$; compare Fig. 4(b). At this angle, the absorption lines SW-25 to SW-22 shift down in frequency by $0.2 \mathrm{GHz}$. The up-bending of SW-23 occurs already for fields of $-23 \mathrm{mT}$ and SW-24 appears in connection with SW-23 in the field region of the up-bending. Up to angles of $35^{\circ}$ as shown in Fig. 4(c) the modes SW-25 to SW-23 resume to shift down by $0.3 \mathrm{GHz}$ while the field dispersion of the mode SW-22 stays the same. The up-bending of SW-23 is smoother and begins already at $-45 \mathrm{mT}$. SW-23 and SW-25 meet at $0 \mathrm{mT}$ and share the same frequency until $12 \mathrm{mT}$. SW-24 is already visible at $-39 \mathrm{mT}$. At $40^{\circ}$ (not shown) the mode SW-23 shifts up

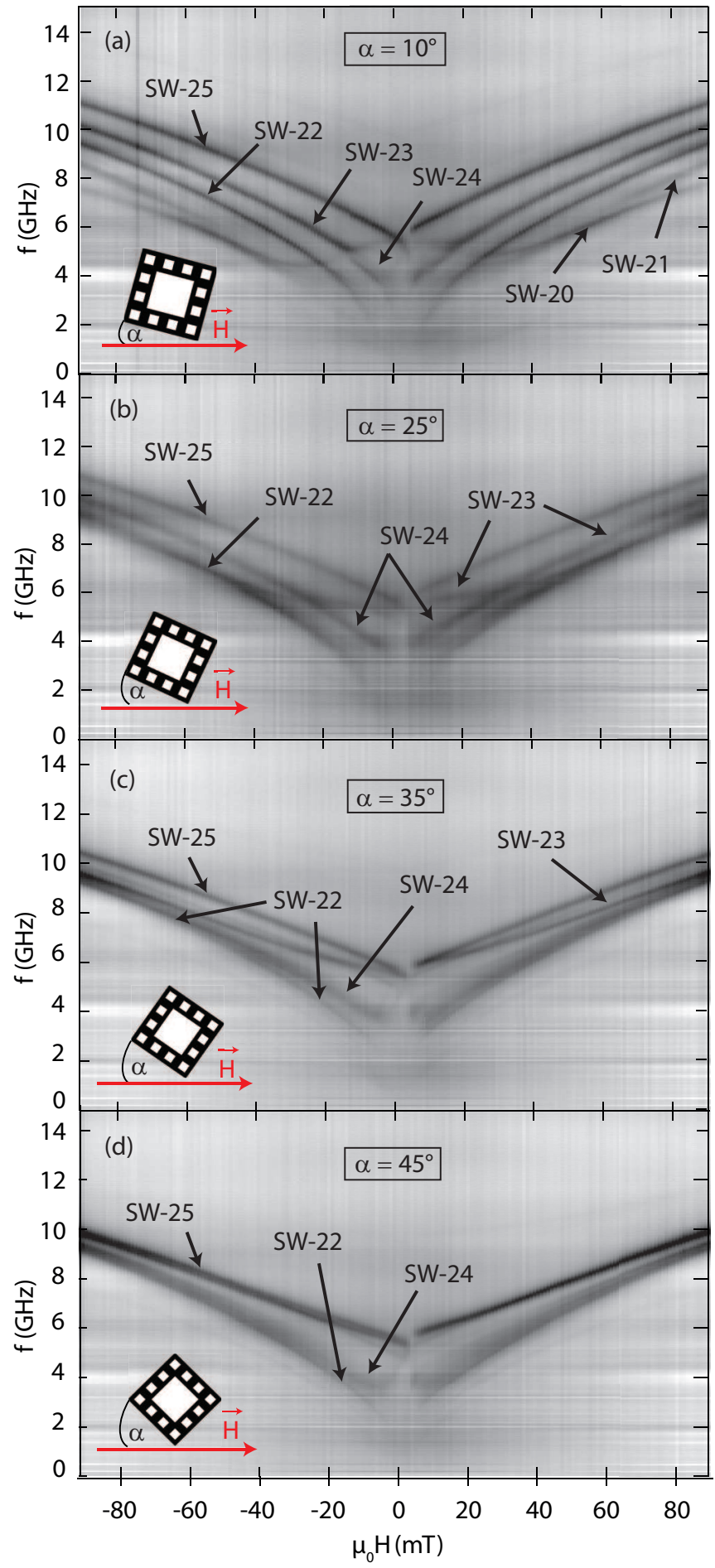

FIG. 4. (Color online) Transmission spectra of Sierpinski carpets $\mathrm{SC}(4,12,2)$ where the sides of the fractals are rotated with respect to the external field vector $\vec{H}$ by the angle $\alpha$ as mimicked by the insets.

by $0.3 \mathrm{GHz}$ while no considerable changes are observed for the remaining absorption lines. For $45^{\circ}$, however, only three absorption lines are visible; compare Fig. 4(d). While the absorption line SW-23 is no longer visible in the spectra, SW-25 shifts down by $0.5 \mathrm{GHz}$ and shows twice the intensity as for smaller angles. The absorption lines of SW-22 and SW-24 smear out and the spectra show no sharp absorption 
lines in the branch between $9.7 \mathrm{GHz}$ and $2.8 \mathrm{GHz}$. The modes in this frequency range are not clearly distinguishable.

We conclude that the spin-wave spectra depend crucially on the relative orientation of external field vector and the sides of the Sierpinski carpets. By increasing the enclosing angle $\alpha$ the number of distinct spin-wave modes decreases in the spectra. For a parallel alignment of the field and the diagonal of the fractals at $\alpha=45^{\circ}$ spin-wave excitation is observed in two broad frequency bands. Variation of the angle $\alpha$ enables us to control the complexity of the magnetization pattern and with it the excitation as well as the frequency of spin-wave modes in Sierpinski carpets, as discussed in detail in Sec. V. Spin-wave spectra in Sierpinski carpets can be tuned by their magnetic configuration besides geometrical parameters such as iteration level and fractal dimension.

\section{MICROMAGNETIC SIMULATIONS}

In order to correlate the measured field-dispersion relations with the spatial localization of the individual spinwave modes, we performed micromagnetic simulations using the MicroMagnum code [41]. Computational capacities dictate a down-scaling of the sizes of the fractals to $3.5 \mu \mathrm{m} \times 3.5 \mu \mathrm{m} \times 30 \mathrm{~nm}$. The discretization cell size is $5 \times 5 \times 30 \mathrm{~nm}^{3}$, in the range of the exchange length $l_{\mathrm{ex}}=\sqrt{2 A / \mu_{0} M_{\mathrm{S}}^{2}}=5.3 \mathrm{~nm}$ of the soft magnetic Permalloy where $\mu_{0}=4 \pi \times 10^{-7} \mathrm{H} / \mathrm{m}$ is the vacuum permeability. With $A=13 \times 10^{-12} \mathrm{~J} / \mathrm{m}$ for the exchange constant, $M_{\mathrm{S}}=860 \mathrm{kA} / \mathrm{m}$ for the saturation magnetization, and $\alpha=0.01$ for Gilbert damping, typical parameters $[34,42,43]$ for Permalloy are used. After simulating the field hysteresis of the relaxed magnetization along the $x$ axis between $-90 \mathrm{mT}$ and $90 \mathrm{mT}$, a field pulse is applied in the $y z$ plane of the structures for the dynamic simulations. The field vector is slightly tilted away from the $x$ axis in order to break the symmetry and to obtain a stable magnetization configuration. The spatially homogeneous pulse has a Gaussian time modulation with a full width at half maximum of $3 \mathrm{ps}$ [44] and an amplitude of $1 \mathrm{mT}$. The simulation data are obtained for a time period of $20 \mathrm{~ns}$ with a time resolution of $25 \mathrm{ps}$. The spectral distribution as well as the spatially resolved magnitude and phase of magnetization precession are obtained by applying fast Fourier transformation (FFT) to the out-of-plane component of the magnetization in each cell. Due to the small tilting of the field, we expect the spatially resolved magnitude to be point symmetric about the center of the carpets. Simulations for the fractal iteration levels $i=0$ to 2 are presented.

\section{DISCUSSION}

Figure 5 shows the simulated spectra as well as the spatially resolved magnitude of magnetization precession of individual spin-wave modes for Sierpinski carpets $\mathrm{SC}(4,12,0)$ and $\operatorname{SC}(4,12,1)$. The field vector is aligned parallel to the side of the carpets; i.e., $\alpha=0^{\circ}$. The simulated spectra of solid squares, i.e., $i=0$, show a similar field dispersion of the mode SW-01 and its higher-order modes to that of the experimental spectra [45]; compare Figs. 2(a) and 5(a). Between $-1 \mathrm{mT}$ and $-3 \mathrm{mT}$ the simulations did not yield a stable magnetization
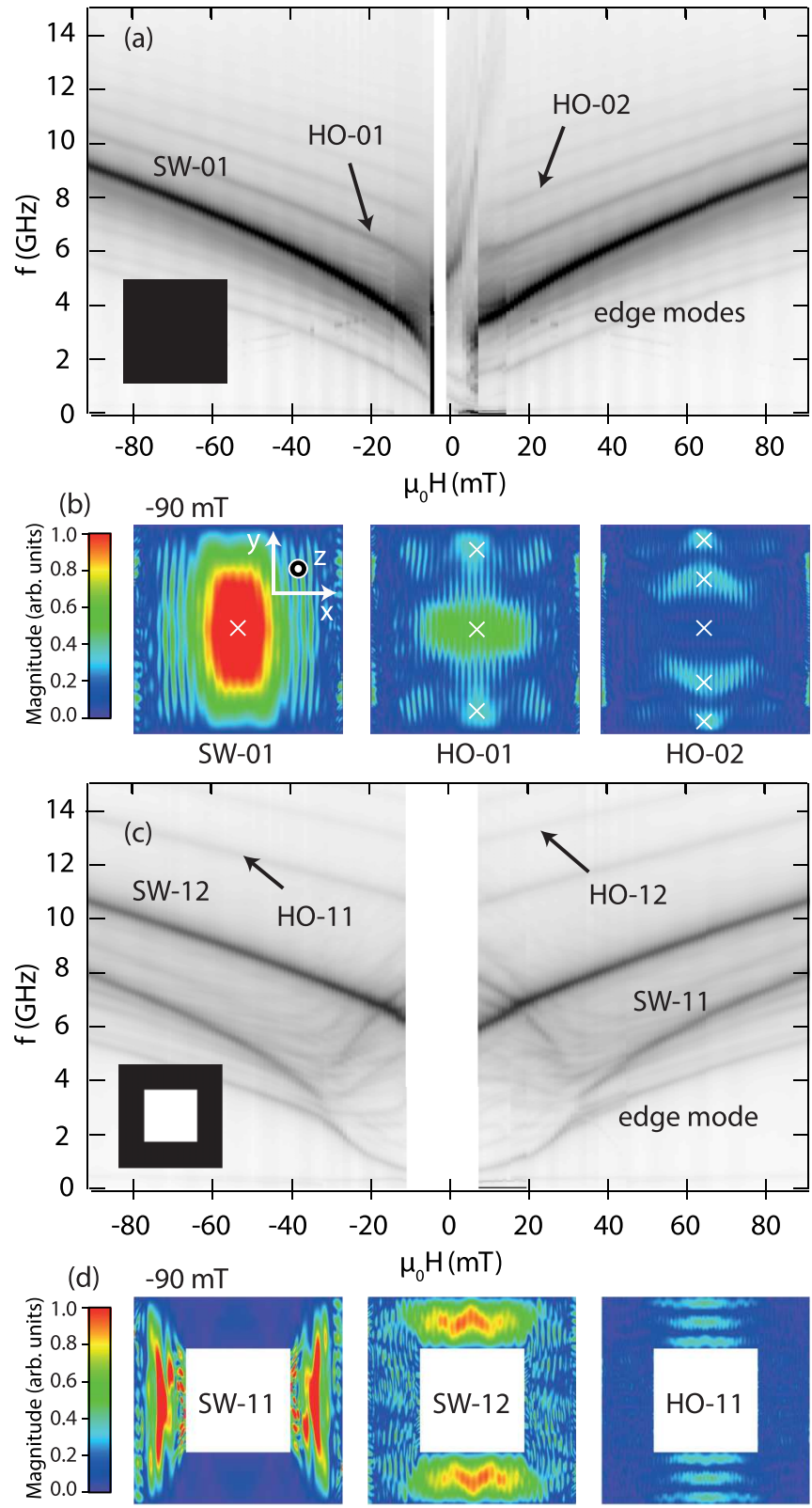

FIG. 5. (Color online) (a) Simulated field dependence of the resonant spin-wave modes of a Sierpinski carpet $\mathrm{SC}(4,12,0)$. The gray scale encodes the averaged magnitude of the Fourier-transformed pulse response in arbitrary units. (b) Spatially resolved magnitude of the magnetization precession at resonance in $\mathrm{SC}(4,12,0)$ for a field strength of $\mu_{0} H=-90 \mathrm{mT}$. The antinodes of the spin-wave modes are marked by the white crosses. (c) Field dependence of the resonance frequencies of a Sierpinski carpet SC $(4,12,1)$. (d) Spatially resolved magnitude of the magnetization precession at resonance in $\mathrm{SC}(4,12,1)$ for a field strength of $\mu_{0} H=-90 \mathrm{mT}$.

configuration after application of the field pulse. Differences in frequencies and switching fields originate from the smaller lateral sizes of the simulated structures. The spatially resolved magnitudes of magnetization precession in Fig. 5(b) show that the mode SW-01 is a dipole-dominated spin-wave mode with one antinode [46] $(l=0$ and $m=0)$ located in the center of the structure. For the higher-order modes HO-01 and HO-02 three $(l=0$ and $m=2)$ and five $(l=0$ and $m=4)$ antinodes are 
visible, respectively. The quantization in the $y$ direction reveals the DE character of the mode SW-01 and its higher-order modes, since the magnetization is aligned along the $x$ axis. The observations comply with studies of the spin-wave spectra of rectangular elements with comparable in-plane sizes $[10,11]$. The frequency of the mode SW-01 is decreasing with magnetic field strength in accordance with the dispersion relation of $\mathrm{DE}$ surface modes [37].

For the iteration level $i=1$, the simulated spectra show the absorption lines of the modes SW-11 and SW-12 as well as the higher-order modes HO-11 and HO-12 and are similar to the corresponding experimental spectra; compare Figs. 2(b) and 5(c). The mode profile is related to that of antidot arrays [17,47] and square nanorings [48]. No stable magnetization configuration was obtained in the simulations between $-10 \mathrm{mT}$ and $7 \mathrm{mT}$. In order to analyze the correlation of field dispersion and localization of the individual modes, it is convenient to divide the fractal $\operatorname{SC}(4,12,1)$ into the rectangles $\mathrm{A}$ [subsquares 2,3 and 10,11 in Fig. 1(c)] and B [subsquares 5, 7 and 6, 8 in Fig. 1(c)]. The subsquares 1, 4, 9, and 12 are shared one-half each by A and B. For the rectangles $\mathrm{A}$, the magnetization is aligned parallel to the easy axis in the $x$ direction and is supposed to switch within a few milliteslas during reversal of the external field. In contrast, the magnetization in rectangles $\mathrm{B}$ is aligned along the hard axis in the $x$ direction for high field strengths. For smaller fields, the magnetization slowly turns towards the easy axis in the $y$ direction. Since higher field strengths are required to align the magnetization along the hard axis than along the easy axis, the switching of the magnetization expands over a larger field regime for rectangles B. The spatially resolved magnitude of precession in Fig. 5(d) shows that the mode SW-12 and its higher-order modes HO-11 and HO-12 are located in rectangles A and have a DE character, since the wave vector is quantized along the $y$ direction perpendicular to the magnetization. The mode SW-11 is located in rectangles $\mathrm{B}$ with its wave vector along the $x$ direction. For high field strengths, the mode SW-11 has a $\mathrm{BV}$ character with a parallel alignment of wave vector and magnetization. In the experimental spectra in Fig. 2(b), the magnetization in rectangles B starts to turn towards the easy axis at a field strength of $-12 \mathrm{mT}$. This is visible by a decrease in the intensity of the absorption line since magnetization and exciting field $\vec{H}_{\mathrm{rf}}$ are not aligned perpendicularly anymore. For $-6 \mathrm{mT}$, the magnetization is predominantly aligned along the easy axis and the mode loses its pure BV character and a $\mathrm{DE}$ character is introduced. This is visible in the spectra by an abrupt increase in frequency caused by the different dispersion relations of $\mathrm{DE}$ and $\mathrm{BV}$ modes [36,49]. Between $-2 \mathrm{mT}$ and $6 \mathrm{mT}$ the magnetization is completely aligned along the easy axis and $\vec{H}_{\mathrm{rf}}$ and no spin waves are excited. The widths of rectangles $\mathrm{A}$ and $\mathrm{B}$ decrease with the fractal dimension and the switching of the magnetization is determined by shape anisotropy. For $\mathrm{SC}(3,8,1)$ and $\mathrm{SC}(4,12,1)$ the absorption line SW-12 is symmetric about the switching field range similar to the characteristics of solid squares in Fig. 2(a). In contrast, the discontinuity of SW-12 for the smallest fractal dimension $\mathrm{SC}(5,16,1)$ resembles switching characteristics of longitudinally magnetized long rectangles $[34,35]$. The up-
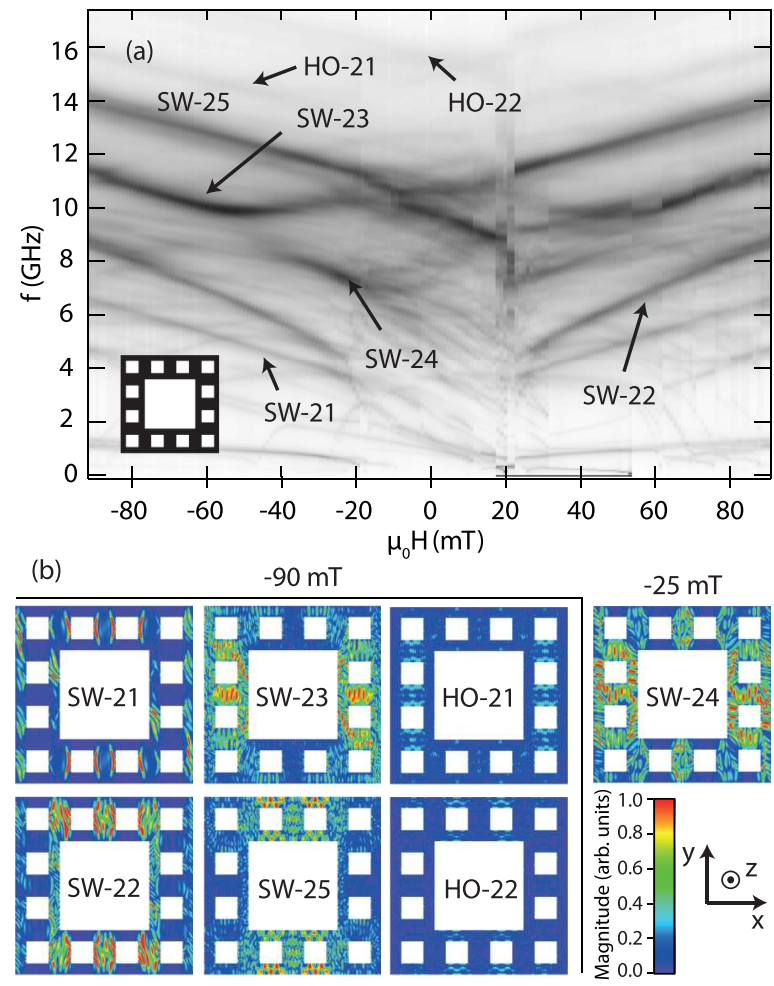

FIG. 6. (Color online) (a) Simulated field dependence of the resonant modes of a Sierpinski carpet $\mathrm{SC}(4,12,2)$. (b) Spatially resolved magnitude of the magnetization precession at resonance for selected field strengths.

bending of SW-11 occurs within wider field ranges and shifts to higher field strengths with decreasing fractal dimension. For the highest fractal dimension $\mathrm{SC}(3,8,1)$, the magnetization in rectangles $\mathrm{B}$ is aligned along the weakly defined hard axis and turns towards the easy axis at $-3 \mathrm{mT}$ within $2 \mathrm{mT}$ before the absorption line SW-11 disappears; compare Fig. 3(a). For the lowest fractal dimension $\operatorname{SC}(5,16,1)$, the magnetization turns towards the strongly defined hard axis already at $-8 \mathrm{mT}$ within a range of $6 \mathrm{mT}$; compare Fig. 3(b).

The simulated field distribution of the spin-wave modes for a Sierpinski carpet $\operatorname{SC}(4,12,2)$ is shown in Fig. 6(a). The five absorption lines SW-21 to SW-25 as well as the higher-order modes HO-21 and HO-22 can be identified by comparing the simulated spectra with the experimental results in Fig. 2(c). The spatially resolved magnitudes of precession in Fig. 6(b) show the localization of the modes within the carpet. SW-21 is an exchange-dominated mode strongly localized at the edges of the holes that were introduced in this segmentation step. At the edges, the internal magnetic field is expected to be significantly reduced in direction of the applied field $[10,11]$. The mode SW-22 in between the holes in rectangles $\mathrm{A}$ is weaker localized and has a stronger dipole dominated character. SW-23 and its higher-order mode HO-21 are dipoledominated DE modes located between the holes in rectangles $\mathrm{B}$ where a homogeneous internal field is expected. Their wave vectors are quantized along the $y$ direction perpendicularly to the magnetization. This configuration is shared by the DE modes SW-25 and HO-22 that can be found above and below 

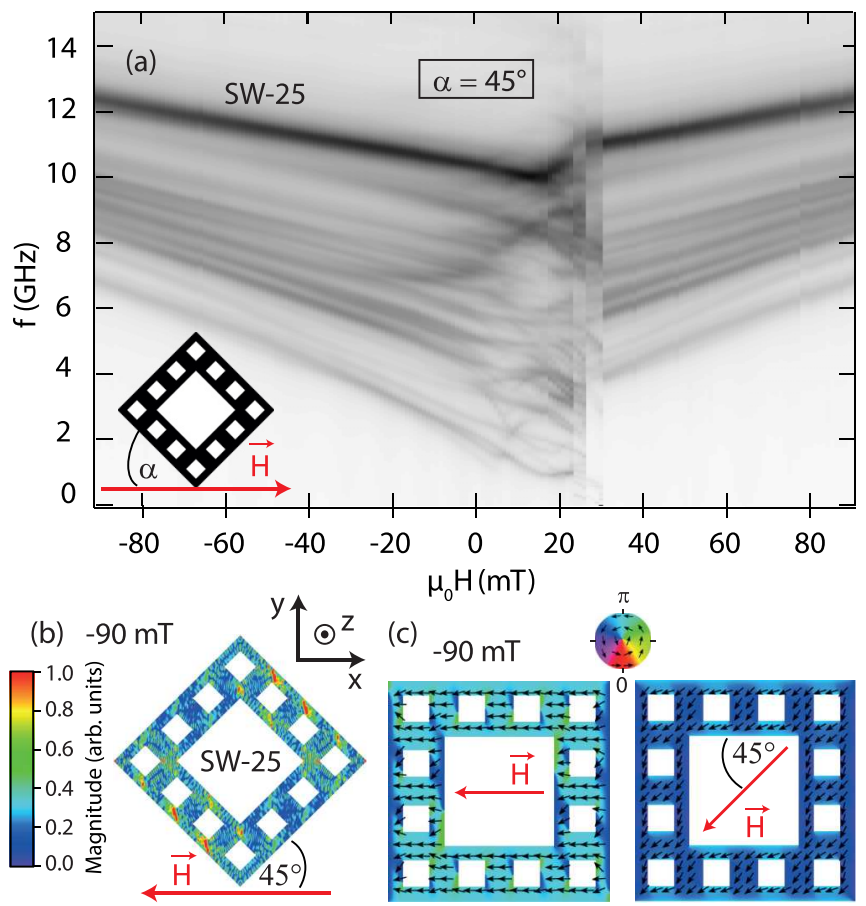

FIG. 7. (Color online) (a) Simulated field dependence of the resonance frequencies of a Sierpinski carpet $\mathrm{SC}(4,12,2)$ where the sides of the fractal are rotated relatively to the external field vector $\vec{H}$ by the angle $\alpha=45^{\circ}$. (b) Spatially resolved magnitude of the magnetization precession at resonance for a field strength of $-90 \mathrm{mT}$. (c) Simulated static magnetization of $\operatorname{SC}(4,12,2)$ for $\alpha=0^{\circ}$ and $45^{\circ}$. The color scale displays the alignment of the magnetization relative to the magnetic field along the positive $x$ direction.

the holes in rectangles A. Similarly to the experimental spectra, SW-23 bends up at $-41 \mathrm{mT}$, disappears as well as HO-21 at $-28 \mathrm{mT}$, before the mode SW-24 appears at $-27 \mathrm{mT}$. The latter is predominantly located in rectangles B with its magnitude distributed uniformly around the holes. The origin of the up-bending of SW-23 may be attributed to frequency gaps in the dispersion relation of the spin-wave modes but cannot be clarified completely. For fields between $-6 \mathrm{mT}$ and $20 \mathrm{mT}$, the simulated static magnetization is rather unstable and the pulse excitation leads to a complex mode spectrum during magnetization reversal. For higher fields, a symmetric mode spectrum for positive and negative field directions can be observed.

Further simulations were performed for Sierpinski carpets $\mathrm{SC}(3,8, i)$ and $\mathrm{SC}(5,16, i)$ at $i=1,2$ and the corresponding modes SW-21 to SW-25 as well as SW-11 and SW-12 could be identified in the spectra (results not shown). The spatially resolved magnitude of precession shows that the localization of the individual modes is similar to that for $\operatorname{SC}(4,12, i)$ and justifies the same notation for all three fractal dimensions. In accordance with the experimental results, the frequency splitting between individual modes increases with decreasing fractal dimension.

Figure 7(a) shows simulated data for a Sierpinski carpet $\mathrm{SC}(4,12,2)$ where the external field is rotated with respect to the sides of the fractal by an angle of $\alpha=45^{\circ}$. The field distribution shows one absorption line with a high intensity and a branch consisting of several nearby low-intensity absorption lines. The field distribution is similar to the experimental spectra in Fig. 4(d) and the high-intensity mode can be identified as SW-25. The spatially resolved magnitude of precession in Fig. 7(b) shows that the mode SW-25 is unlocalized and distributed over the whole fractal. When turning the magnetic field vector towards the diagonal of the fractal, the orientations of the magnetization in rectangles $\mathrm{A}$ and $\mathrm{B}$ assimilate. As a consequence, the dipole-dominated modes SW-23 and SW-25 combine to one spin-wave mode for angles $\alpha>35^{\circ}$ as observed in the experimental data in Figs. 4(c) and 4(d). The simulated static magnetizations for the angles $\alpha=0^{\circ}$ and $45^{\circ}$ are shown in Fig. 7(c). The inhomogeneity of the internal magnetic field is significantly reduced when the external field is aligned along the diagonal, since the magnetization meets the edges of the fractal with an angle of $45^{\circ}$ instead of $90^{\circ}$. Thus, the excitation of the exchange-dominated modes SW-21 and SW-22 localized at edges, where the inhomogeneity of the internal field is high, is almost completely suppressed for $\alpha>35^{\circ}$.

\section{CONCLUSION}

We have studied spin-wave spectra of Permalloy Sierpinski carpets by experiments and micromagnetic simulations. The number of quantized spin-wave modes in the spectra increases with the iteration level and the accompanying inhomogeneity of the internal field of the carpets. The frequency splitting between the modes increases with decreasing fractal dimension in agreement with the theory in Ref. [29]. Independently of these two geometrical parameters the number and frequencies of the spin-wave modes are controlled by tuning the complexity of the magnetization patterns of the Sierpinski carpets via the external magnetic field. The wave characteristics and the localization of the spin-wave modes are identified by the simulated spatially resolved magnitude of magnetization precession.

Ferromagnetic Sierpinski carpets are potential candidates for magnonics as high-frequency multiband devices whose frequency characteristics can be tuned both by geometrical structuring and their magnetic configuration.

\section{ACKNOWLEDGMENTS}

We thank Ulrich Merkt, Lars Bocklage, and Max Hänze for fruitful discussions, André Drews and Theo Gerhardt for valuable advice on the micromagnetic simulations with MicroMagnum, Jan Michael Möller for support during sample preparation, and Michael Volkmann for excellent technical assistance. Financial support of the Deutsche Forschungsgemeinschaft via Sonderforschungsbereich 668 "Magnetism from the Single Atom to the Nanostructure" and Graduiertenkolleg 1286 "Functional Metal-Semiconductor Hybrid Systems" is gratefully acknowledged. This work has been supported by the excellence cluster "The Hamburg Centre for Ultrafast Imaging Structure, Dynamics, and Control of Matter on the Atomic Scale" of the Deutsche Forschungsgemeinschaft. 
[1] R. Hertel, W. Wulfhekel, and J. Kirschner, Phys. Rev. Lett. 93, 257202 (2004).

[2] S. V. Vasiliev, V. V. Kruglyak, M. L. Sokolovskii, and A. N. Kuchko, J. Appl. Phys. 101, 113919 (2007).

[3] K.-S. Lee and S.-K. Kim, J. Appl. Phys. 104, 053909 (2008).

[4] T. Schneider, A. A. Serga, B. Leven, B. Hillebrands, R. L. Stamps, and M. Kostylev, Appl. Phys. Lett. 92, 022505 (2008).

[5] C. Mathieu, J. Jorzick, A. Frank, S. O. Demokritov, A. N. Slavin, B. Hillebrands, B. Bartenlian, C. Chappert, D. Decanini, F. Rousseaux, and E. Cambril, Phys. Rev. Lett. 81, 3968 (1998).

[6] J. Jorzick, S. O. Demokritov, B. Hillebrands, M. Bailleul, C. Fermon, K. Y. Guslienko, A. N. Slavin, D. V. Berkov, and N. L. Gorn, Phys. Rev. Lett. 88, 047204 (2002).

[7] K. Y. Guslienko, S. O. Demokritov, B. Hillebrands, and A. N. Slavin, Phys. Rev. B 66, 132402 (2002).

[8] J. P. Park, P. Eames, D. M. Engebretson, J. Berezovsky, and P. A. Crowell, Phys. Rev. Lett. 89, 277201 (2002).

[9] J. P. Park, P. Eames, D. M. Engebretson, J. Berezovsky, and P. A. Crowell, Phys. Rev. B 67, 020403 (2003).

[10] K. Y. Guslienko, R. W. Chantrell, and A. N. Slavin, Phys. Rev. B 68, 024422 (2003).

[11] C. Bayer, J. Jorzick, B. Hillebrands, S. O. Demokritov, R. Kouba, R. Bozinoski, A. N. Slavin, K. Y. Guslienko, D. V. Berkov, N. L. Gorn, and M. P. Kostylev, Phys. Rev. B 72, 064427 (2005).

[12] M. Krawczyk and D. Grundler, J. Phys.: Condens. Matter 26, 123202 (2014).

[13] B. Lenk, H. Ulrichs, F. Garbs, and M. Münzenberg, Phys. Rep. 507, 107 (2011).

[14] J. Topp, D. Heitmann, M. P. Kostylev, and D. Grundler, Phys. Rev. Lett. 104, 207205 (2010).

[15] A. V. Chumak, A. A. Serga, B. Hillebrands, and M. P. Kostylev, Appl. Phys. Lett. 93, 022508 (2008).

[16] S. Schnittger, S. Dreyer, C. Jooss, S. Sievers, and U. Siegner, Appl. Phys. Lett. 90, 042506 (2007).

[17] S. Martens, O. Albrecht, K. Nielsch, and D. Görlitz, J. Appl. Phys. 105, 07C113 (2009).

[18] G. Meier, M. Kleiber, D. Grundler, D. Heitmann, and R. Wiesendanger, Appl. Phys. Lett. 72, 2168 (1998).

[19] Z. K. Wang, V. L. Zhang, H. S. Lim, S. C. Ng, M. H. Kuok, S. Jain, and A. O. Adeyeye, Appl. Phys. Lett. 94, 083112 (2009).

[20] S.-K. Kim, J. Phys. D: Appl. Phys. 43, 264004 (2010).

[21] M. Mruczkiewicz, M. Krawczyk, V. K. Sakharov, Y. V. Khivintsev, Y. A. Filimonov, and S. A. Nikitov, J. Appl. Phys. 113, 093908 (2013).

[22] B. B. Mandelbrot, Fractals: Form, Chance, and Dimension (Freeman, San Francisco, 1977).

[23] F. Hausdorff, Math. Ann. 79, 157 (1918).

[24] H. Brune, Surf. Sci. Rep. 31, 125 (1998).

[25] S. Alexander, C. Laermans, R. Orbach, and H. M. Rosenberg, Phys. Rev. B 28, 4615 (1983).
[26] T. Nakayama, K. Yakubo, and R. L. Orbach, Rev. Mod. Phys. 66, 381 (1994).

[27] M. Kohmoto, B. Sutherland, and C. Tang, Phys. Rev. B 35, 1020 (1987).

[28] C. Puente-Baliarda, J. Romeu, R. Pous, and A. Cardama, IEEE Trans. Antennas Propag. 46, 517 (1998).

[29] P. Monceau and J.-C. S. Lévy, Phys. Lett. A 374, 1872 (2010).

[30] P. Monceau and J.-C. S. Lévy, Physica E 44, 1697 (2012).

[31] Note that a solid square which is divided into $n^{2}$ subsquares features $p=n^{2}$ self-similar pieces and an integer dimension of $d=\ln \left(n^{2}\right) / \ln (n)=2$.

[32] A. Vogel, A. Drews, T. Kamionka, M. Bolte, and G. Meier, Phys. Rev. Lett. 105, 037201 (2010).

[33] A. Vogel, A. Drews, M. Weigand, and G. Meier, AIP Adv. 02, 042180 (2012).

[34] C. Swoboda, N. Kuhlmann, M. Martens, A. Vogel, and G. Meier, J. Appl. Phys. 114, 043905 (2013).

[35] N. Kuhlmann, A. Vogel, and G. Meier, Phys. Rev. B 85, 014410 (2012).

[36] S. O. Demokritov, B. Hillebrands, and A. N. Slavin, Phys. Rep. 348, 441 (2001).

[37] R. W. Damon and J. R. Eshbach, J. Phys. Chem. Solids 19, 308 (1961).

[38] P. H. Bryant, J. F. Smyth, S. Schultz, and D. R. Fredkin, Phys. Rev. B 47, 11255 (1993).

[39] K. Vogt, H. Schultheiss, S. Jain, J. E. Pearson, A. Hoffmann, S. D. Bader, and B. Hillebrands, Appl. Phys. Lett. 101, 042410 (2012).

[40] L. Bocklage, S. Motl-Ziegler, J. Topp, T. Matsuyama, and G. Meier, J. Phys.: Condens. Matter 26, 266003 (2014).

[41] MicroMagnum, http://micromagnum.informatik.uni-hamburg. de.

[42] G. Nahrwold, J. M. Scholtyssek, S. Motl-Ziegler, O. Albrecht, U. Merkt, and G. Meier, J. Appl. Phys. 108, 013907 (2010).

[43] A. Drews, B. Krüger, M. Bolte, and G. Meier, Phys. Rev. B 77, 094413 (2008).

[44] This pulse duration length guarantees frequency components far beyond the considered frequency range.

[45] Note that the absorption lines in the experimentally measured normalized transmission of Fig. 2 are proportional to the averaged squared magnitude of precession whereas the simulated spectra show the averaged FFT amplitudes.

[46] For reasons of visibility we count the number of antinodes here instead of the number of nodes.

[47] R. Mandal, S. Saha, D. Kumar, S. Barman, S. Pal, K. Das, A. K. Raychaudhuri, Y. Fukuma, Y. Otani, and A. Barman, ACS Nano 6, 3397 (2012).

[48] C. Banerjee, S. Saha, S. Barman, O. Rousseau, Y. Otani, and A. Barman, J. Appl. Phys. 116, 163912 (2014).

[49] B. A. Kalinikos and A. N. Slavin, J. Phys. C: Solid State Phys. 19, 7013 (1986). 